\section{Craniofacial surgery for orbital malformations}

year in England and Wales. Usually diagnosed at birth, the underlying aetiology is of premature fusion of one or more of the cranial sutures. This results in restriction of growth in a direction perpendicular to the orientation of the fused suture, and failure of expansion of the skull in that area in response to enlargement of the underlying growing brain. Despite this, the brain continues to expand, the expansion being displaced into areas where the remaining cranial sutures are patent. This results not only in restriction of growth in the area of the affected suture, but also in compensatory overgrowth in areas of the skull distant from the prematurely fused suture, giving rise to abnormalities of both shape and volume of the skull. Each fused suture will give rise to a characteristic skull shape, and the presence of the characteristic shape on clinical examination indicates the diagnosis (Table 2). Restriction of skull volume may result in raised intracranial pressure (ICP) in $10-15 \%$ of patients with single suture synostosis, ${ }^{3}$ and a higher incidence with multiple suture involvement.

A number of studies have examined ocular and orbital problems in nonsyndromic craniosynostosis, with significant risks of astigmatism, strabismus, ptosis, lateral canthal dystopia, nasolacrimal obstruction, and other ocular problems. ${ }^{4-6}$

Surgical management is indicated for two reasons. Growth restriction in the presence of a rapidly expanding growing brain may result in raised ICP, and surgery may be indicated for the prevention or treatment of raised ICP. The second indication is cosmetic, to avoid future psychological and social problems as a result of abnormal appearance. ${ }^{8,9}$ The principals of surgical management include (1) excision of the affected suture, thus releasing the growth restriction, (2) normalisation of abnormal shape by remodelling the existing deformed area of the skull, and (3) correction of the cranial volume deficit by repositioning the re-modelled bone to increase intracranial volume and allow

\section{Nonsyndromic craniosynostoses}

The incidence of nonsyndromic craniosynostosis is approximately 1 in $2500,,^{1,2}$ generating approximately $200-240$ cases per 
normal expansion of the underlying brain. For those conditions affecting the forehead and orbital roof, this is achieved by means of frontoorbital advancement and re-modelling. A bicoronal flap is raised exposing the frontal bones and upper half of the orbits. A bifrontal craniotomy is carried out, followed by removal of the supraorbital bar of bone, which includes the upper half of the orbital rim and the anterior part of the orbital roof. The abnormally shaped bone is then re-modelled by means of multiple osteotomies to achieve a normal shape, and it is then replaced in a much more advanced position to bring the forehead and supraorbital region well in front of the anterior surface of the globe. This usually leaves a large bony gap at the posterior edge of the re-modelled segment, but this fills in with new bone as the pericranium and dura possess significant osteogenic potential, as surgery is carried out during infancy (variable timing, usually at 12-18 months of age for frontal deformity). Occasionally, frontoorbital advancement and re-modelling is carried out in older patients where the original deformity has re-emerged as a result of re-fusion of the affected suture (very uncommon in nonsyndromic craniosynostosis) or owing to poor subsequent growth. ${ }^{10}$

\section{Syndromic craniosynostoses}

Syndromic craniosynostosis refers to a group of patients in which craniosynostosis is associated with other abnormalities, often affecting the orbits and midface. ${ }^{11}$

Table 1 The craniofacial team

\begin{tabular}{lll}
\hline Surgical & Nonsurgical & Others \\
\hline Anaesthesia/ITU & Genetics & $\begin{array}{l}\text { Cleft lip and } \\
\text { palate team }\end{array}$ \\
& & Dentist \\
ENT & Orthodontics & Neurology \\
Maxillofacial & Speech and language & Oncology \\
Neurosurgery & Psychology & Radiology \\
Ophthalmology & & Coordinator \\
Plastic surgery & & \\
\hline
\end{tabular}

These are much more rare than nonsyndromic cases, comprising approximately $10 \%$ of the craniosynostosis population. In these patients, craniosynostosis is usually associated with hypoplasia of the midface, (eg Crouzons syndrome), and in addition there may be other (often limb) abnormalities (eg Aperts syndrome). The principal of abnormal skull shapes and volumes in nonsyndromic patients applies not only to the skull of syndromic patients but also the bony orbits and midface, because of the more extensive midface abnormalities in the syndromic population. The bony orbit is shallow owing to retrusion of both the supraorbital and infraorbital margins, and compensatory bulging of the temporal lobe into the posterolateral wall of the orbits further reduces the volume over an already shallow orbit, resulting in ocular proptosis. On occasions this can be very severe. Hypertelorism and antimongloid slant of the palpable fissure may also be present. High incidences of strabismus and amblyopia reflective error and astigmatism have been reported.

There is a three-dimensional reduction in volume of the bones of the midface, and this can result in significant upper airway obstruction sometimes requiring tracheotomy, problems with middle ear aeration, and dentofacial deformity.

Surgical management of syndromic craniosynostosis is much more complex than in the nonsyndromic patients. Syndromic craniosynostosis patients require frontoorbital advancement and re-modelling, and correction of the midface abnormalities. This may include midface advancement, correction of hypertelorism, and other adjunctive procedures such as augmentation rhinoplasty, repair of associated cleft palate, orthodontic treatment, etc. Frontoorbital advancement is often carried out in the same way as the nonsyndromic population, but may be indicated at the same time as midface advancement (for instance, for severe proptosis) in a so-called monoblock ${ }^{12}$ procedure. Correction of midface hypoplasia is based on the Le Fort III osteotomy, which separates the whole of the midfacial skeleton, including the zygomas, lower half of orbit, nose, and maxilla from the skull base, allowing

Table 2 Craniosynostoses

\begin{tabular}{|c|c|c|}
\hline Suture & Shape of skull & Descriptive term \\
\hline Sagittal suture & Long and narrow & Scaphocephaly \\
\hline Metopic suture & Triangular skull with pointed forehead & Trigonocephaly \\
\hline Uni coronal & $\begin{array}{l}\text { Twisted skull with unilateral forehead } \\
\text { and orbital recession }\end{array}$ & Frontal plagiocephaly \\
\hline Bicoronal & Flat forehead wide skull & Frontal brachycephaly \\
\hline Uni lamboid & Unilateral posterior flattening & $\begin{array}{l}\text { Occipital plagiocepahly (rare-usually } \\
\text { deformational) }\end{array}$ \\
\hline Bilateral lamboid & Posterior flattening & Posterior brachycephaly \\
\hline All sutures & Severe deformity with bitemporal bulging & Clover leaf skull \\
\hline
\end{tabular}


repositioning in a more anterior and sometime inferior position in order to correct the periorbital and midface anomalies. For a number of reasons, the movement of the midface is carried out slowly by gradual traction applied using internal or external distraction devices (or a combination of both) with movements at $1 \mathrm{~mm} /$ day over several weeks. This has the advantage of allowing gradual soft tissue adaptation to bony re-positioning and creating new bone in the osteotomy gap by distraction osteogenesis. Midface advancement is usually carried out in later childhood to minimise the operative risk (particularly bleeding), which can be difficult to control.

Where midface retrusion is associated with hypertelorism (usually in Aperts syndrome), a variation of Le Fort III osteotomy known as Facial Bipartition ${ }^{13}$ can correct the interorbital distance as well as the anteroposterior and vertical midface hypoplasia. This involves removal of a triangular segment of bone from the midline of the osteotomised midface, with its base at the supraorbital bar, and its apex between the upper incisor teeth. Removal of the segment of bone allows medial rotation of the right and left halves of the midface, reducing the interorbital distance. It also has the effect of widening the narrow maxilla and levelling out what is often a V-shaped configuration of the upper teeth. Having reduced the interorbital distance during surgery, the whole midface segment is then moved by gradual distraction osteogenesis to correct the anteroposterior and vertical deficiencies. The aim of midface advancement is to normalise the relationship of the orbital rim to the eye. In doing this, the zygomas, nose, and maxilla are also improved. However, complete correction of the nasomaxillary deficiency often requires more advancement than would be desirable at the orbital margins, and this can result in enophthalmos. Both the nose and maxilla are often deficient vertically, and full correction requires a vertical elongation of the midface, a movement that can further increase orbital volume and contribute to postoperative enophthalmos. The maxillary deficiency may therefore not be fully corrected by Le Fort III osteotomy, and further osteotomy (often Le Fort I) may be required later to address this. ${ }^{14}$ In addition, midface advancement is usually carried out during childhood when further growth of both the maxilla and mandible are to be expected. This further growth in the maxilla is likely to be deficient as a result of the underlying syndrome, whereas mandibular growth proceeds in a relatively normal manner. This often results in a worsening of the jaw relationship with time, leading to a recurrence of the deformity in the lower part of the face (class III jaw relationship or relative mandibular prognathism) and a requirement for a further (maxillary) osteotomy at Le Fort I level when growth has finished. This clearly illustrates some of the difficulties encountered in these patients, and the limitations of some of the surgical techniques available.

A variety of less extensive osteotomies are available for use in selected cases. In milder cases of midface hypoplasia where maxillary hypoplasia is associated with infraorbital flattening, a Kufner variant of the Le Fort III osteotomy can be useful. This effectively brings infraorbital margins and maxilla forward when the forehead, malar, and nasal projections are satisfactory.

\section{Orbital malposition}

Hypertelorism, telecanthus, and orbital dystopia can occur as isolated conditions, or may be associated with facial clefting, encephalocoele, sinonasal pathology, or trauma. ${ }^{11}$ Conditions in which there is underdevelopment of nasal projection may give an appearance or illusion of hypertelorism (eg Larsen syndrome, Binder syndrome).

In these cases, nasal reconstruction giving adequate nasal projection will correct the apparent deformity, and may give a perceptual improvement in cases of mild hypertelorism where major surgery is not indicated. However, for most significant orbital malformations, some orbital osteotomy will be required. ${ }^{15,16}$ A variety of orbital osteotomies have been described, which may involve movement of one, two, three, or four orbital walls, unilaterally or bilaterally depending on the severity and nature of the deformity. ${ }^{17,18}$

Of paramount importance in orbital osteotomies is the management of the medial canthal ligament. This is best left attached to its bony base and great care must be taken in subperiosteal dissection to avoid encroaching on the area of the medical canthus. If detachment of the medial canthus is unavoidable or occurs inadvertently, then careful transnasal medial canthopexy is necessary, with bone grafting required in order to re-position the canthus and give it a secure attachment. However, results of medial canthopexy can be disappointing, and is best avoided by maintaining the attachment of the medial canthal ligament intact. Bone grafting is always required in orbital osteotomies to fill bony gaps left by the orbital movements and to ensure postoperative stability. Subsequent secondary procedures are sometimes required for the management of associated facial deformity or postoperative ocular problems. ${ }^{11}$

\section{References}

1 Kirby PJ, Beverly DW, Batchelor AG. Frequency of craniosynostosis in Yorkshire, UK. Lancet 1993; 341(8857): 1412-1413.

2 French LR, Jackson IT, Melton III LJ. A population based study of craniosynostosis. J Clin Epidemiol 1990; 43(1): 69-73. 
3 Thompson DN, Malcolm GP, Jones BM, Harkness WJ, Hayward RD. Intracranial pressure in single suture craniosynostosis. Paediatr Neurosurg 1995; 22: 235.

4 Denis D, Genitori L, Bardot J, Saracco JB, Choux M, Maumenee IH. Ocular findings in trigonocephaly. Graefes Arch Clin Exp Ophthalmol 1994; 232(12): 728-733.

5 Denis D, Genitori L, Conrath J, Lena G, Choux M. Ocular findings in children operated on for plagiocephaly and trigonocephaly. Childs Nerv Syst 1996; 12(11): 683-689.

6 Gray TL, Casey T, Selva D, Anderson PJ, David DJ. Ophthalmic sequelae of Crouzon syndrome. Ophthalmology 2005; 112(6): 1129-1134.

7 Gault DT, Renier D, Marchac D, Jones BM. Intracranial pressure and intracranial volume in children with craniosynostosis. Plast Reconstr Surg 1992; 90: 377-381.

8 Endriga MC, Kapp-Simon KA. Psychological issues in craniofacial care: state of the art. Cleft Palate Craniofac J 1999; 36(1): 3-11.

9 Speltz ML, Morton K, Goodell EW, Clarren SK. Psychological functioning of children with craniofacial anomalies and their mothers: follow-up from late infancy to school entry. Cleft Palate Craniofac J 1993; 30(5): 482-489.

10 Reinhart E, Muhling J, Michel C, Collmann H, Pistner H, Reuther J. Craniofacial growth characteristics after bilateral fronto-orbital advancement in children with premature craniosynostosis. Childs Nrev Syst 1996; 12(11): 690-694.
11 Lin KY, Ogle RC, Jane JA. Craniofacial Surgery - Science and Surgical Technique. WB Saunders Co: Philadelphia, 2002, pp 332-344.

12 Nishimoto S, Oyama T, Shimizu F, Tsugawa T, Nagashima $\mathrm{T}$, Yamamoto $\mathrm{K}$ et al. Fronto-facial monobloc advancement with rigid external distraction (RED-II) system. J Cranciofac Surg 2004; 15(1): 54-59.

13 Posnick JC. Monobloc and facial bipartition osteotomies: a step-by-step description of the surgical technique. J Craniofac Surg 1996; 7(3): 229-250.

14 Satoh K, Mitsukawa N, Hosaka Y. Dual midfacial distraction osteogenesis: Le Fort III minus I and Le Fort I for syndromic craniosynostosis. Plast Reconstr Surg 2003; 111(3): 1019-1028.

15 Tessier P, Guiot G, Derome P. Orbital hypertelorism. II. Definite treatment of orbital hypertelorism by craniofacial or by extracranial osteotomies. Scand J Plast Reconstr Surg 1973; 7(1): 39-58.

16 Ortiz-Monasterio F, Molina F. Orbital hypertelorism. Clin Plast Surg 1994; 21(4): 599-612.

17 Persing JA, Jane JA, Park TS, Edgerton MT, Delashaw JB. Floating C-shaped orbital osteotomy for orbital rim advancement in craniosynostosis: preliminary report. J Neurosurg 1990; 72(1): 22-26.

18 Psillakis JM, Zanini SA, Godoy R, Cardim VL. Orbital hypertelorism: modification of the craniofacial osteotomy line. J Maxillofac Surg 1981; 9(1): 10-14. 